\title{
A Scaling Approach to Ideal Quantum Gases
}

\author{
Thomas Nattermann* \\ Institut für Theoretische Physik, Universität zu Köln, 50937 Köln, Germany
}

(Dated: August 1, 2021)

\begin{abstract}
The thermodynamic properties of ideal quantum gases are derived solely from dimensional arguments, the Pauli principle and thermodynamic relations, without resorting to statistical mechanics.
\end{abstract}

PACS numbers:

\section{INTRODUCTION}

In this article we present a derivation of the thermal equations of state of ideal quantum gases by using solely dimensional analysis, thermodynamic considerations and simple physical arguments 1 . The thermodynamic relations of gases depend strongly on the energymomentum relation of the gas particles and on their spin, i.e., whether the particles are fermions or bosons. The energy-momentum relation of free massive particles is given by

$$
E_{\mathbf{p}}=\sqrt{m^{2} c^{4}+c^{2} \mathbf{p}^{2}}-m c^{2}
$$

$m$ denotes the rest mass of the particles, $c$ is the velocity of light. Here we have subtracted the rest energy $m c^{2}$ from the particle energy which results in a redefinition of the chemical potential, i.e. $\mu$ has to be replaced by $\mu-m c^{2}$ further on. In the non-relativistic limit $m c \gg|\mathbf{p}|$, $E_{\mathbf{p}} \approx \frac{\mathbf{p}^{2}}{2 m}$, whereas in the ultra-relativistic limit $m c \ll$ $|\mathbf{p}|, E_{\mathbf{p}} \approx c|\mathbf{p}|$. Both cases can be written in the single exponent dispersion relation

$$
E_{\mathbf{p}} \sim m c^{2}\left(\frac{|\mathbf{p}|}{m c}\right)^{\nu} \sim \gamma_{\nu}|\mathbf{p}|^{\nu}, \quad \gamma_{\nu}=m^{1-\nu} c^{2-\nu},
$$

where $\nu=1,2$ for relativistic and non-relativistic particles, respectively.

Another group of particles to which our considerations apply are quasi-particles in condensed matter systems. In these systems deviations from the state of perfect order can be described by a superposition of quantized elementary excitations which behave as bosonic particles with an energy momentum relation also of the form (1.2). The quasi-particles we are considering, as well as photons, have a vanishing chemical potential, i.e. their number is not fixed. At low temperatures this number is small and hence their interaction can be neglected. Quasi-particles are characterized by a fixed value of $\nu>0$ 2. $\gamma_{\nu}$ has to be expressed in terms of the quasi-particle parameters. Examples for the case $\nu=1$ are lattice vibration of solids with acoustic phonons as quasi-particles 3 ( $c$ denotes now the sound velocity) or spin waves in anti-ferromagnets with magnons as quasi-particles ${ }^{4}$ ( $c$ denotes here the spin

*natter@thp.uni-koeln.de wave velocity). An example with $\nu=2$ are magnons in ferromagnets ${ }^{5}$, where $m \sim \hbar^{2} /\left(b^{2} T_{c}\right)$. $b$ denotes the lattice constant and $T_{c}$ the Curie temperature. For further examples of quasi-particles see e.g. reference ${ }^{6}$.

It is interesting to remark that our considerations can be extended to non-integer values of $\nu$. One example are capillary wave excitations of the surface of superfluid ${ }^{4} \mathrm{He}$, the so-called ripplons ${ }^{7}$ which show a dispersion relation (1.2) with $m / c \sim \rho \hbar / \sigma$ and $\nu=3 / 2$. Here $\sigma$ and $\rho$ denote surface tension and the liquid density, respectively. Our results apply also to this case, but in the further discussion we will mainly focus on the cases of integer values of $\nu$.

\section{DIMENSIONAL ANALYSIS}

\section{A. The classical case}

Considering a homogeneous gas of $N$ point particles confined in a container of volume $V=v N$ and coupled to a bath of temperature $T$, the free energy can be written in the form

$$
F(T, V, N)=N F(T, v, 1)=N f(T, v) .
$$

$f(T, v)$ is the free energy per particle. The mean separation $a$ between particles is related to the volume per particle $v=a^{d}$, where $d$ denotes the dimension of the system. The pressure follows then from $p=-(\partial f / \partial v)_{T}$.

We begin with the consideration of a classical gas with fixed exponent $\nu$ of the dispersion relation (1.2). In this case the pressure can only depend on the three parameters $\gamma_{\nu}, a$ and $T$. There is no further parameter. The temperature $T$ appears in thermodynamics in the relation $T^{-1}=\partial S / \partial E$, where $S$ and $E$ denote the entropy and the energy, respectively. Entropy is determined only up to a multiplicative (Boltzmann) $\operatorname{constant}^{8}$. For the moment we will only assume that this constant is dimensionless, i.e. we measure $T$ in energy units.

Denoting the dimension of mass, length and time by $M, L$ and $t$, respectively, the dimension of $\gamma_{\nu}, a, T$ and $p$ are $M^{1-\nu} L^{2-\nu} t^{\nu-2}, L, M L^{2} t^{-2}$ and $M L^{2-d} t^{-2}$, respectively. Since the three parameters have different dimensions, there is a unique combination of these which has the dimension of pressure $\frac{9}{}$

$$
p \sim \frac{T}{a^{d}}, \quad a^{d}=v=\frac{V}{N},
$$


as expected. We fix now our temperature scale by choosing the constant of proportionality in (2.4) to be equal one, i.e. $p v=T$, which is the known result ${ }^{10}$.

This suggest $f(T, v) \sim T \ln v$. Clearly $v$ should occur in the free energy in combination with another volume to make the argument of the logarithm dimensionless. This shows the limitations of classical physics.

In the case of massive particles there is only a dependence on $m$ or $c$ as long as we use the single power law relation (1.2) with $\nu=1$ or 2 fixed. However, for the description of the cross-over between the nonrelativistic and the relativistic regime both $m$ and $c$ occur and hence a new dimensionless parameter $T /\left(m c^{2}\right)$ appears. The relation (2.4) is formally correct even in the region $T \gg m c^{2}$. However, one has to expect that in this region pair creation processes will take place and a grand canonical description is more appropriate, as we will consider in section

In the case of charged particles the ideal gas properties are only preserved under certain conditions. If we consider for simplicity electrons (on a neutralizing positively charged background) the electron-electron interaction is of the order $e^{2} / a$. To have still an ideal gas the Coulomb energy has to be small compared with the thermal energy, i.e. $e^{2} / a \ll T$ or $a_{T}=e^{2} / T \ll a \underline{11}$.

\section{B. Quantum case}

In the quantum case the Planck constant of action $\hbar$ appears as a new parameter. The pressure is now no longer uniquely fixed by dimensional analysis since in order to determine the four exponents of $\gamma_{\nu}, a, T$ and $\hbar$ only three relations (from the comparison of the powers of $t$, $L$ and $M$ ) exist. Thus the equation of state will depend additionally on a dimensionsless parameter $x_{\nu}$ which includes $\hbar$ as well as $T$, $a$ and $\gamma_{\nu}$. Dimensional analysis shows that the only possible choice is ${ }^{12}$

$$
\begin{gathered}
x_{\nu}=\frac{a}{\hbar}\left(\frac{T}{\gamma_{\nu}}\right)^{1 / \nu}=: \frac{a}{\lambda_{T, \nu}}, \\
\lambda_{T, \nu}=\hbar\left(\frac{\gamma_{\nu}}{T}\right)^{1 / \nu}=\star_{C}\left(\frac{m c^{2}}{T}\right)^{1 / \nu}, \star_{C}=\frac{\hbar}{m c} .
\end{gathered}
$$

Here $\lambda_{T, \nu}$ denotes the thermal de Broglie wave length of the (quasi-) particles. $\lambda_{C}$ is the Compton wave length in the case of massive particles. We ignore all numerical pre-factors since they are beyond the accuracy of the dimensional analysis presented here. Since for $\hbar \rightarrow 0$, i.e. $\lambda_{T, \nu} \ll a$, we have to regain the classical result (2.4), quantum behavior will be seen for $x_{\nu} \lesssim 1$, i.e. for $a \lesssim \lambda_{T, \nu}$. The borderline $x_{\nu} \approx 1$ defines a characteristic energy $\gamma_{\nu}(\hbar / a)^{\nu}$ which turns out to be the Fermi energy for fermions and the temperature of Bose condensation for bosons, respectively. This will be discussed in detail in the subsequent section.

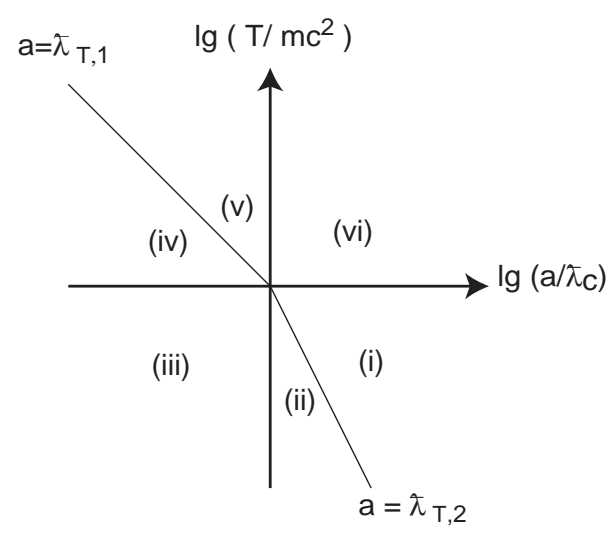

FIG. 1: The various regions (i) - (iv) of the parameter space of massive particles depicted in the $\log \left(T / m c^{2}\right)$ versus $\log \left(a / \star_{C}\right)$ plane. Massive matter under ordinary conditions $\left(T \ll m c^{2}\right)$ is located in a region well below the horizontal axis. For more explanations see the text.

In addition to the cross-over from the classical to the quantum regime, massive particles also show a crossover from non-relativistic to relativistic behavior. If the mean particle distance is given by $a$, then for $a \gg \lambda_{C}$, the typical particle energy $E_{|\mathbf{p}| \sim \hbar / a}$ approaches the nonrelativistic expression $\hbar^{2} /\left(m a^{2}\right)$ whereas for $a \ll \lambda_{C}$ the relativistic expression $c \hbar / a$ applies.

From the mutual order of the three length scales $a, \star_{C}$ and $\lambda_{T, \nu}$ six regions can be distinguished ${ }^{13}$. Anticipating the result of the detailed discussions of the following sections these six regions exhibit very different physical properties :

(i) $\lambda_{C}<\lambda_{T, 2}<a$ : This is the region of classical behavior. The only relevant length scale is the the inter-particle distance $a$.

(ii) $\lambda_{C}<a<\lambda_{T, 2}$ : The region of non-relativistic quantum behavior. Fermions are degenerated, bosons condense. Since $\lambda_{C}<a, \lambda_{T, \nu}$ relativistic effects do not play a role.

(iii) $a<\lambda_{C}<\lambda_{T, 2}$ : $\mathrm{A}$ region of quantum behavior where bosons behave non-relativistically (as in (iii)) whereas fermions exhibit relativistic effects. The Pauli principle makes the difference.

(iv) $a<\lambda_{T, 1}<\lambda_{C}$ : Fermions show still low temperature behavior (as in (iii)), bosons exhibit particleantiparticle pair production.

(v) $\lambda_{T, 1}<a<\lambda_{C}$ : Fermions and bosons are described by the same equation of state as photons.

(vi) $\lambda_{T, 1}<\lambda_{C}<a$ : Same behavior as in (v).

Bose condensation as well as particle-antiparticle pair production will in general change the effective interparticle distance from $a$ to $\lambda_{T, 2}$ and $\lambda_{T, 1}$, respectively. 
Since pair production keeps some of the quantum numbers constant (e.g. the total charge or the lepton or hadron number) in these regions $a$ is a measure of the corresponding quantum number of the gas.

For charged fermions dimensional analysis gives a further intrinsic length scale, the Bohr radius $a_{\mathrm{Bohr}}=$ $\hbar^{2} /\left(m e^{2}\right)$. For simplicity we consider again a gas of electrons on a positively charged background. To have still an ideal gas the Coulomb interaction $\sim e^{2} / a$ has to be small compared to the kinetic energy. For non-relativistic electrons this results in $e^{2} / a \ll \hbar^{2} /\left(m a^{2}\right)$, i.e., $a \ll$ $\hbar^{2} /\left(m e^{2}\right)=a_{\text {Bohr }}$. For relativistic electrons one obtains in the same way $e^{2} / a \ll \hbar c / a$ or $e^{2} /(\hbar c) \approx 1 / 137 \ll 1$, i.e., the ideal gas condition is always fulfilled. For nonrelativistic charged bosons outside the condensate, $a$ has to be replaced by $\lambda_{T, 2}$ (see below) which leads to the condition $\lambda_{T, 2}<a_{\text {Bohr }}$. The effect of Coulomb interaction will be more pronounced in the condensate since the kinetic energy is zero. Indeed, it has been argued that there is no Bose condensation in the presence of Coulomb interaction ${ }^{14}$.

The different regions of the parameter space can be visualized in the diagram depicted in Figure 1 where we plot $T / m c^{2}=\left(\lambda_{C} / \lambda_{T, \nu}\right)^{\nu}$ versus $a / \lambda_{C}$ in a double logarithmic representation. The lines $T=m c^{2}$ and $a=\lambda_{C}$ correspond to the horizontal and vertical axis, respectively. The line $a=\lambda_{T, \nu}$ separates the classical region $a>\lambda_{T, \nu}$ from the quantum region $a<\lambda_{T, \nu}$. In our double logarithmic plot this line is straight with a slope equal to $-\nu$. Ordinary massive matter at temperatures realized on earth is restricted to the region below the horizontal axis. For electrons $m_{e} c^{2} \sim 10^{10} \mathrm{~K}$, and hence for room temperatures $T \sim 10^{2} \mathrm{~K}$ this region is given by $T / m c^{2} \leq 10^{-8}$. The Bohr radius $a_{B}$ corresponds to $a_{B} / \lambda_{C}=\hbar c / e^{2} \approx 137$.

\section{Grand Canonical Description}

So far we assumed that the particle number $N=V / a^{d}$ and hence $a$ was fixed. In some cases it is more appropriate to consider the grand canonical description where instead of $N$ the chemical potential $\mu$ is given. This description is in particular useful in those parameter regions where particle creation and annihilation processes (like the inverse $\beta$-decay or electron-positron pair creation) become important.

The corresponding thermodynamic potential $J(T, V, \mu)$ is related to the pressure by the GibbsDuham relation

$$
J(T, V, \mu)=V J(T, 1, \mu) \equiv V j(T, \mu)=-p V .
$$

Since $T$ and $\mu$ have the same dimension there is no intrinsic length scale and hence no purely classical expression for the pressure. If we include $\hbar$ as a further parameter

$$
\star_{T, \nu} \omega\left(\frac{\mu}{T}\right)
$$

appears as the only length scale of the problem. $\omega\left(\frac{\mu}{T}\right)$ is so far an arbitrary but dimensionless function of $\mu / T$. Following our previous dimensional arguments the pressure can now be written as 15.16

$$
p=\frac{T}{\star_{T, \nu}^{d}} \Omega\left(\frac{\mu}{T}\right) .
$$

with $\Omega\left(\frac{\mu}{T}\right)$ likewise so far unknown. For particles with vanishing chemical potential the pressure is always given by equation (2.9) with $\Omega(0) \sim 1$. Important examples are photons, magnons in anti-ferromagnets and acoustical phonons (all $\nu=1)$, magnons in ferromagnets $(\nu=2)$ and ripplons $(\nu=3 / 2)$.

With (2.7) we get $J=-T \Omega\left(\frac{\mu}{T}\right) V \star_{T, \nu}^{-d}$ and hence

$$
\frac{\partial J}{\partial \mu}=-N=-\frac{V}{\lambda_{T, \nu}^{d}} \Omega^{\prime}\left(\frac{\mu}{T}\right)=-\frac{p V}{T} \frac{\Omega^{\prime}}{\Omega}
$$

Using the classical equation of state (2.4) this requires $d \Omega_{c l} / d x=\Omega_{c l}$. Hence for a classical gas

$$
\Omega_{c l}\left(\frac{\mu}{T}\right)=\Omega_{c l}(0) e^{\mu / T} .
$$

(2.9) and (2.11) can be used to calculate the chemical potential of the classical gas $\frac{17}{17}$

$$
\mu=T \ln \left(\frac{p \star_{T, \nu}^{d}}{T \Omega_{c l}(0)}\right)=T \ln \left(\frac{\lambda_{T, \nu}^{d}}{a^{d} \Omega_{c l}(0)}\right) .
$$

\section{EQUATION OF STATE}

\section{A. Weak quantum corrections}

Since for $x_{\nu} \rightarrow \infty$ the system has to approach the classical limit we make the following Ansatz for the pressure

$$
p=\frac{T}{a^{d}} \psi_{\mathrm{F} / \mathrm{B}}\left(x_{\nu}\right)
$$

with $\psi_{\mathrm{F} / \mathrm{B}}\left(x_{\nu} \rightarrow \infty\right) \rightarrow$ const. Here the subscript $\mathrm{F}$ or $\mathrm{B}$ stands for fermions or bosons. The scaling function $\psi_{\mathrm{F} / \mathrm{B}}\left(x_{\nu}\right)$ describes the cross-over from classical to quantum behavior of particles with a dispersion relation with fixed value of $\nu$. For massive particles this Ansatz can be generalized to

$$
p=\frac{T}{a^{d}} \Psi_{\mathrm{F} / \mathrm{B}}\left(x_{1}, \frac{a}{\lambda_{C}},\right)
$$

which describes in addition also the cross-over from nonrelativistic to ultra-relativistic behavior. The scaling function $\Psi_{\mathrm{F} / \mathrm{B}}\left(x_{1}, y\right)$ can be related to (3.13) in the limiting cases $y \rightarrow \infty$ and $y \rightarrow 0$, respectively. In the nonrelativistic limit $a \gg \star_{C}$, i.e. $y \rightarrow \infty, \Psi_{\mathrm{F} / \mathrm{B}}\left(x_{1}, y\right) \sim$ $\psi_{\mathrm{F} / \mathrm{B}}\left(\sqrt{x_{1} y}\right)=\psi_{\mathrm{F} / \mathrm{B}}\left(x_{2}\right)$. In the ultra-relativistic case 
$a \ll \lambda_{C}$, i.e. $y \rightarrow 0, \Psi_{\mathrm{F} / \mathrm{B}}\left(x_{1}, y\right) \sim \psi_{\mathrm{F} / \mathrm{B}}\left(x_{1}\right)$. In the following we will mainly concentrate on the discussion of $\psi_{\mathrm{F} / \mathrm{B}}\left(x_{\nu}\right)$.

We consider first the case of weak quantum fluctuations. Assuming that the pressure can be expanded in powers of the particle density, i.e. in powers of $x_{\nu}^{-d}$, (the classical result (2.4) is the lowest order term of this expansion) $\psi_{\mathrm{F} / \mathrm{B}}\left(x_{\nu}\right)$ can be written in the form

$$
\psi_{F / B}\left(x_{\nu}\right) \approx 1 \pm \text { const. } x_{\nu}^{-d}, \quad x_{\nu} \gg 1
$$

Here the plus and minus sign corresponds to fermions and bosons, respectively. Indeed, for fermions the Pauli principle will lead to an increase of the pressure with respect to the classical case. For bosons the pressure is reduced since quantum mechanics increases the probability for the double occupancy of a state ${ }^{18}$. 2.5), (3.13) and (3.15) describe indeed the lowest order quantum correction to the classical equation of state $\frac{19}{}$.

\section{B. Strong quantum limit - fermions}

In the strong quantum limit $x_{\nu} \rightarrow 0$, we have even more pronounced differences between fermions and bosons. For fermions the Pauli-principle guarantees a non-zero pressure even for $T \rightarrow 0$. From (2.5) and (3.13) this requires $\psi_{F}\left(x_{\nu}\right) \sim x_{\nu}^{-\nu}$ and hence

$$
p_{F}(\nu) \sim \frac{\hbar^{\nu} \gamma_{\nu}}{a^{d+\nu}} \sim \frac{E_{F, \nu}(a)}{a^{d}} .
$$

On the r.h.s. we have introduced the Fermi energy

$$
E_{F, \nu}(a) \sim \frac{\hbar^{\nu} \gamma_{\nu}}{a^{\nu}}
$$

which is the kinetic energy of the fermions with momentum $|\mathbf{p}| \sim \hbar / a$. Apparently, in (3.16) the Fermi energy replaces the temperatures of the classical expression (2.4). In particular this gives for relativistic particles ${ }^{20}$

$$
p_{\mathrm{F}}(\nu=1) \sim \frac{\hbar c}{a^{d+1}},
$$

and for non-relativistic particles 21

$$
p_{\mathrm{F}}(\nu=2) \sim \frac{\hbar^{2}}{m a^{d+2}},
$$

respectively. Both cases can be combined in the scaling formula

$$
p_{\mathrm{F}} \sim \frac{\hbar c}{a^{d+1}} \frac{\Psi_{\mathrm{F}}\left(x_{1}, \frac{a}{\bar{\hbar}_{C}}\right)}{\psi_{F}\left(x_{1}\right)} .
$$

The precise form of the scaling function $\Psi_{\mathrm{F}}(x, y)$ remains unknown in this approach. The cross-over between (3.19) and (3.18) takes place at $a \sim \lambda_{C}$, as follows from the general properties of $\Psi_{\mathrm{F}}(x, y)$ discussed before.
As long as $\lambda_{C}<a<\lambda_{T, 2}$ (region (ii) in Figure 1) the particles show non-relativistic quantum behavior. This situation exists e.g. for electrons in solids $\left(a \sim 10^{-10} \mathrm{~m}, \lambda_{C} \sim 10^{-13} \mathrm{~m}\right)$ or neutrons in neutron stars $\left(a \sim 10^{-13} \mathrm{~m}, \lambda_{C} \sim 10^{-16} \mathrm{~m}\right)$.

On the other hand, for $a<\lambda_{C}$ the kinetic energy is larger than $m c^{2}$ and the particles behave relativistically. One has to expect that this 'low temperature' relativistic quantum behavior is seen as long as the temperature is small compared to the Fermi energy $E_{F, \nu}(a) \sim c \hbar / a$, i.e. for $a \lesssim \lambda_{T, 1}$ ( corresponding to regions (iii) and (iv) of Figure 1). Relativistic electrons exist e.g. in white dwarfs $\left(a \sim 10^{-13} \mathrm{~m}\right)$.

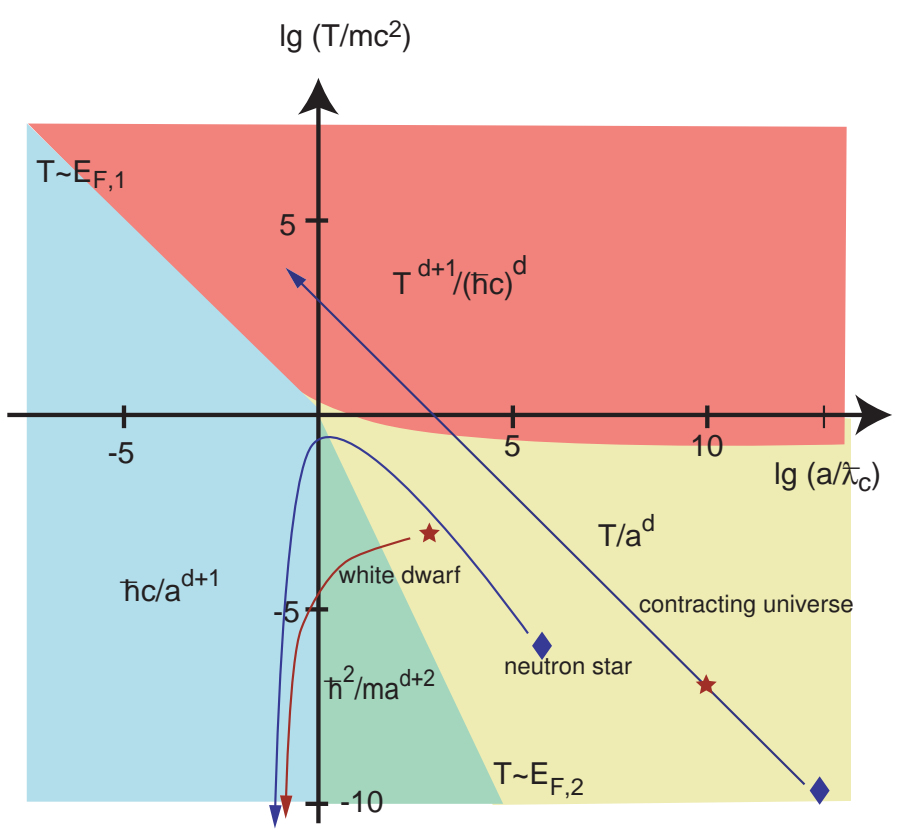

FIG. 2: The pressure of an ideal gas of massive fermions in different parameter regions. The cross-over lines $T=E_{F, 2}$ and $T=E_{F, 1}$ separate the classical $\left(p \sim T a^{-d}\right)$ from the non-relativistic quantum region $\left(p \sim \hbar^{2} /\left(m a^{d+2}\right)\right.$ and the radiation $\left(p \sim T^{d+1} /(\hbar c)^{d}\right)$ from relativistic quantum region $\left(p \sim \hbar c / a^{d+1}\right)$, respectively. $E_{F, \nu}(a)$ denotes the Fermi energy. The vertical line $a=\lambda_{C}, T<m c^{2}$ separates the non-relativistic from the relativistic quantum behavior. The radiation region is characterized by pair creation processes where the effective particle distance is $\lambda_{T, 1}<a$. The symbols $\downarrow$ and $\star$ stand for the protons and electrons, respectively, which change their coordinates in this figure during the evolution of stars and the toy universe, respectively, considered in section IV.

For very dense systems of atoms, where nuclei and electrons form a plasma with $a \ll a_{\text {Bohr }}$, electrons and protons may recombine to produce a neutron and a neutrino (the inverse $\beta$-decay). The charge of the resulting nucleus is then reduced by one. In this case the electron density is no longer an independent variable but determined by the chemical potential $\tilde{\mu}$ of this process. If $T \ll \tilde{\mu}$ and initially $a<\lambda_{C}$ the system is in the ultrarelativistic region, thermal fluctuations can be neglected. 
$\tilde{\mu}$ determines then unambigously a new length scale

$$
\lambda_{\mu}=\frac{\hbar c}{\tilde{\mu}}
$$

corresponding to $\omega(\mu / T) \sim T / \mu$ in (2.8) which replaces $a$ in the region $a<\lambda_{\mu}<\lambda_{T, 1}$ in eq.3.18. Since thermal fluctuations are irrelevant and $a$ is not longer fixed, $T$ is replaced by $\tilde{\mu}$ in all expressions. The pressure is therefore reduced to 22

$$
p_{F} \sim \frac{\tilde{\mu}^{d+1}}{(\hbar c)^{d}}
$$

We come now to a discussion of the parameter region of ultra-high temperatures of fermionic systems where particle-antiparticle pair production becomes important (regions (v) and (vi) of Figure 1). In this region the particle number is not conserved and it is more adequate to describe the system by specifying a chemical potential, as we have done in subsection [C] For simplicity we assume that only fermions of the same kind (and their corresponding antiparticles) are generated. As discussed in Subsection IIB the Coulomb interaction can be neglected since the effective particle distance turns out to be much smaller than the Bohr radius $a_{B}$. For temperatures $T \gg \mu$ the pressure is determined by (2.9) with $\omega(\mu / T) \sim 1$ and $\nu=1$ (since we are in the relativistic region), i.e. ${ }^{23}$

$$
p_{\mathrm{F}} \sim \frac{T}{\star_{T, 1}^{d}} \sim \frac{T^{d+1}}{(\hbar c)^{d}}
$$

which agrees with the result for photons (compare (2.9)). Hence we will call (3.23) radiation behavior. $\lambda_{T, 1}$ has to be considered as the mean distance between the fermions which is here smaller than $a$ because of pair production. If several kinds of fermions exist each of it gives the same contribution (3.23) to the pressure.

Finally we consider the cross-over between (3.23) and the pressure formulas valid at lower temperature. We begin with the region $\lambda_{C}<a$. Lowering temperature we expect a cross-over to the classical result (2.4). To describe the cross-over we have to understand the contribution of pair production if we raise temperature starting in the classical region. It is therefore convenient to use a grand canonical description also for the description of this cross-over. To make contact with the classical result (2.4), we have to express the chemical potential of particles and anti-particles in terms of the parameters of the system. Particles and anti-particles can annihilate into photons. Since the sum of the chemical potentials is conserved in this reaction ${ }^{24}$ and the chemical potential of photons is zero, we find that the chemical potentials of particle and anti-particles have opposite sign, say $\pm \mu$. From (2.9) and (2.11) we conclude that in the classical region the density of particles and anti-particles, respectively, is given by

$$
\frac{1}{\lambda_{T, 2}^{d}} \exp \left(\frac{ \pm \mu-m c^{2}}{T}\right) \text {. }
$$

Here we have included explicitly the term $-m c^{2}$ resulting from the shift of the energy scale discussed in 【 If the density of generated particles is small compared to the initial density $a^{-d}$ such that $\lambda_{T, 2}^{-d} e^{\left(\mu-m c^{2}\right) /(T)} \approx a^{-d}$ we get after elementary manipulations for the density of the generated particles

$$
\frac{a^{d}}{\lambda_{T, 2}^{2 d}} \exp \left(\frac{-2 m c^{2}}{T}\right) .
$$

The cross-over to the radiation region sets in if the density 3.25 of generated particles is a finite fraction of the initial density $a^{-d}$. This gives for the cross-over line $a_{c o}(T)$ (compare Figure 2)

$$
\frac{a_{c o}}{\lambda_{C}} \sim \sqrt{\frac{m c^{2}}{T}} \exp \left(\frac{m c^{2}}{T d}\right) .
$$

In the relativistic region $a<\lambda_{C}$ essentially the same arguments apply but now we have to replace $\lambda_{T, 2}$ by $\lambda_{T, 1}$ in (3.24). The cross-over follows then at $\lambda_{T, 1} \lesssim$ $a \exp \left(-m c^{2} / T d\right)$ which corresponds for $a<\lambda_{C}$ essentially to $a \sim \lambda_{T, 1}$ (compare Figure 2), in agreement with our previous conjecture.

\section{Strong quantum limit - bosons}

For bosons the low temperature pressure cannot depend on the volume since all bosons may accumulate in the state of lowest energy. This requires in (3.13) $\psi_{B}\left(x_{\nu}\right) \sim x_{\nu}^{d}$ from which we get for the pressure

$$
p_{\mathrm{B}}(\nu) \sim \frac{T}{\lambda_{T, \nu}^{d}} \sim \frac{T^{1+d / \nu}}{\hbar^{d} \gamma_{\nu}^{d / \nu}} \sim\left(\frac{T}{T_{c, \nu}}\right)^{d / \nu} \frac{T}{a^{d}},
$$

where on the r.h.s. we introduced the temperature of Bose condensation

$$
T_{c, \nu}=T_{c, \nu}(a) \sim \frac{\hbar^{\nu} \gamma_{\nu}}{a^{\nu}}
$$

In particular, we get for relativistic particles ${ }^{25}$

$$
p_{\mathrm{B}}(\nu=1) \sim \frac{T}{\lambda_{T, 1}^{d}} \sim \frac{T^{d+1}}{(\hbar c)^{d}}
$$

and for non-relativistic particles 26

$$
p_{\mathrm{B}}(\nu=2) \sim \frac{T}{\lambda_{T, 2}^{d}} \sim \frac{T^{(d+2) / 2}}{\hbar^{d}} m^{d / 2} .
$$


Again both the relativistic and the non-relativistic case can be combined into the scaling form

$$
p_{\mathrm{B}} \sim \frac{T}{\lambda_{T, 1}^{d}} \frac{\Psi_{\mathrm{B}}\left(x_{1}, \frac{a}{\hbar_{C}}\right)}{\psi_{\mathrm{B}}\left(x_{1}\right)} .
$$

The cross-over between (3.30) and (3.29) occurs at $\lambda_{T, 1} \approx$ $\lambda_{C}$, i.e. at $T \approx m c^{2}$.

Since $a<\lambda_{T, \nu}$ the pressure is reduced with respect to the classical result (2.4). This can be explained by assuming that a finite fraction of particles sits now in the state of zero energy and hence does not contribute to the pressure. Comparing (2.4) and (3.27) we conclude that $\lambda_{T, \nu}$ has to be considered as the mean distance of particles above the condensate 27 .

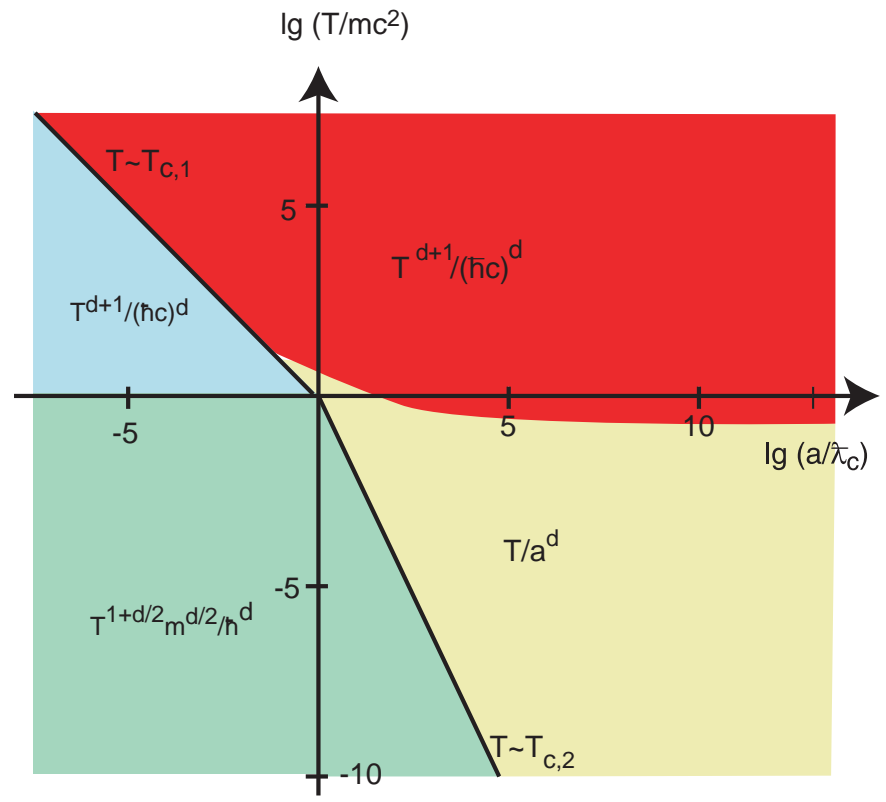

FIG. 3: The pressure of an ideal gas of massive bosons in different parameter regions, as discussed in the text. The line of Bose condensation transition $T_{c, 2}(a)$ in the non-relativistic regime is continued in the relativistic regime as $T_{c, 1}(a)$. There is an additional cross-over at $T \sim m c^{2}$ from non-relativistic to relativistic behavior.

Next we discuss the phase diagram of massive Bose particles obeying the full dispersion relation (1.1). We begin with the classical region $\lambda_{C}<\lambda_{T, 2}<a$ where the pressure is given by (2.4). Reducing $T$ we reach at $a \approx$ $\lambda_{T, 2}$ the region where a macroscopic fraction of particles does not contribute to the pressure since it is in the state of zero energy. As argued above, the number $N_{E>0}$ of the remaining particles of finite energy can be written as

$$
\frac{V}{\lambda_{T, 2}^{d}}=N\left(\frac{a}{\rtimes_{T, 2}}\right)^{d} \sim N\left(\frac{T}{T_{c, 2}}\right)^{d / 2}=N_{E>0} .
$$

Thence the number of particles in the condensate is

$$
N_{0}=N-N_{E>0}=N\left(1-\left(\frac{T}{T_{c, 2}}\right)^{d / 2}\right),
$$

which shows that $T_{c, 2}(a)$ is indeed the transition temperature of Bose condensation ${ }^{28}$. If one enters the condensed phase by lowering $T$ at fixed $a$, the total number of bosons remains fixed whereas the mean distance of the particles above the condensate is equal to $\lambda_{T, 2}(T)$, which increases if we further decrease temperature.

On the other hand, if one approaches the transition line by decreasing $a$ at fixed $T$, the mean distance $\lambda_{T, 2}$ between particles above the condensate remains constant (since $T$ is constant) whereas the excess particles go to the ground state. This behavior is seen also for $a<\lambda_{C}$ since the effective distance of particles contributing to the pressure remains $\lambda_{T, 2}\left(>\lambda_{C}\right)$ (compare Fig.3). Thus, Bose condensation as described by equations (3.30) and (3.33) is seen both in regions (ii) and (iii) of Figure 1.

At sufficiently high temperatures we have to expect that production of particle-antiparticle pairs sets in. For simplicity we will again assume that only bosons of the same type can be created (and their anti-particles). At temperatures $T \gg m c^{2}$ bosons behave relativistically and the pressure is given by (3.29).

The discussion of the cross-over to (2.4) if $a>\lambda_{C}$ and to (3.30) if $a<\lambda_{C}$, respectively, follows essentially the same lines as in the case of fermions, with the important difference that in describing the cross-over in the region $a<\lambda_{T, 2}$ the effective particle distance is now $\lambda_{T, 2}$ (instead of $a$ as in the case of fermions). From (3.25) we therefore conclude that the cross-over occurs at $T \sim m c^{2}$. Note, that the cross-over is smooth, since at $T \approx m c^{2}$, $\lambda_{T, 2} \approx \lambda_{T, 1}$ if $a<\lambda_{C}$.

Since in the relativistic region (iv), $a<\lambda_{T, 1}<\lambda_{C}$, the effective particle density $\lambda_{T, 1}^{-1}$ is smaller than $a^{-d}$ one is inclined to conclude that here both pair creation and Bose condensation takes place. For $a>\lambda_{C}$ the cross-over is the same as for fermions.

\section{ILLUSTRATIONS}

\section{A. Short history of a star}

An important application of the various equations of state can be found in the history of stars which we present here in a cartoon like picture. Initially the star burns hydrogen to helium and other light elements and keeps in this way its temperature $T$. The ratio $T /\left(m_{p} c^{2}\right)$ is of the order $10^{-6}$, where $m_{p}$ is the proton mass. This is the present situation of our sun. The pressure of a star can roughly be described by the classical equation (2.4), which counteracts the gravitational pressure

$$
p_{G} \sim G_{N} \frac{m_{p} M}{a^{3} R} \sim G_{N} \frac{m_{p}^{4 / 3} M^{2 / 3}}{a^{4}},
$$

Here $R$ and $M$ denote the radius and the mass of the star, respectively, and $G_{N}$ is the gravitational constant. We do not present the derivation of (4.34), which is straightforward, but remark that $p_{G} \cdot R^{3}$ is the gravitational energy of a sphere $\sim G_{N} M^{2} / R$, as one obtains also from 
a dimensional argument. The ratio of the mass to the radius of a star in this state is controlled by the thermal equation of state (2.4). Indeed, from the equality of (2.4) and (4.34) follows $M / R \sim T /\left(G_{N} m_{p}\right)$, which increases linearly with temperature.

Once the nuclear fuel is exhausted, temperature cannot be kept and the star collapses under the graviational force. As a result the density increases and the star matter crosses over to the region (ii) of Figure 1, where the pressure is given by (3.19). Since the pressure is now inversely proportional to the mass, mainly the electrons contribute to it. Once under further contraction $a$ has reached $\lambda_{C}$ (corresponding to densities of the order $\sim 10^{7} \mathrm{gcm}^{-3}$ ) (3.18) has to be applied for the pressure. The star has reached the state of a white dwarf (compare Figure 2).

If the density is increased further ( i.e. $a \lesssim \lambda_{\mu}$ ), neutrons are generated via the inverse $\beta$-decay. The pressure is determined then by (3.22) until most of the nucleons became neutrons. For the pressure in neutron stars formulas (3.19) for $a>\lambda_{C}$ and (3.18) for $a<\lambda_{C}$ again apply, but the mass $m_{n} \approx m_{p}$ is now that of the neutrons (compare Figure 2). This is the fate of stars with a mass between 15 and 30 times the mass of the sun.

In the ultra-relativistic regions of white dwarfs and neutrons stars the equality between (4.34) and (3.18) results in a relation for the mass

$$
M_{C}=m_{p}\left(\frac{\hbar c}{G_{N} m_{p}^{2}}\right)^{3 / 2}
$$

This so-called Chandrasekhar mass $M_{C}$ is the maximum mass of white dwarfs and neutron stars. Its value is determined by the thermal equation of state in region (iii) and is of the order of twice the mass of the sun.

\section{B. A toy universe}

As a second illustration let us consider a contracting toy universe. For simplicity, this universe is supposed to have no gravity, i.e. there are no stars, clouds of cosmic dust etc., just photons and diluted massive particles. To make things even simpler the only particles assumed to exist are hydrogen atoms ( a "non-metallic" universe in the language of astronomers). Initially the photon spectrum is assumed to correspond to black body radiation of about $3 \mathrm{~K}$. Since for massless particles $\lambda_{T, 1}$ is the only existing length scale, the typical distance of photons is of the order $\lambda_{T, 1} \sim 10^{-3} \mathrm{~m}$.

The number of H-atoms per $\mathrm{m}^{3}$ is assumed to be of order 1. Hydrogen atoms are bosons and hence could be represented in Figure 3 The horizontal and vertical coordinates of the hydrogen gas in Figure 3 are given by $a_{H} / \lambda_{C, H} \sim 10^{16}$ and $T / m c^{2} \approx 10^{-16}$, respectively, i.e. they are outside of the margins of this figure. $\lambda_{C, H} \sim$ $2.1 \cdot 10^{-16} \mathrm{~m}$ is the Compton wave length of hydrogen.
Next we switch on the cosmic contraction, i.e. we go backwards in time. It is convenient to introduce the contraction factor $r(t)=a(t) / a\left(t_{f}\right)<1$, where $a(t)$ denotes the distance at an earlier time $t<t_{f}$ when the final distance was $a\left(t_{f}\right) \equiv a$. Since contraction of space reduces the wavelength of photons such that $\lambda_{T, 1}(t)=$ $\lambda_{T, 1}\left(t_{f}\right) r(t) \equiv \hbar c / T_{\gamma}(t)$ with $T_{\gamma}(t)=T_{\gamma}\left(t_{f}\right) / r(t)$, i.e. the photon gas heats up.

Without coupling to the photons, the hydrogen atoms form a classical non-relativistic gas (region (i) of Figure 1) which contracts adiabatically. Its temperature $T_{H}(t)=T_{H}\left(t_{f}\right) / r^{2}(t)$ rises proportional to $r^{-2}(t)$, provided there is enough interaction between the hydrogen atoms to reach thermal equilibrium. The horizontal and vertical coordinates of the hydrogen gas in Figure 3 thence change according to $a_{H}(t) / \lambda_{C, p} \sim r(t)$ and $T(t) / m c^{2} \sim r^{-2}(t)$, respectively.

When the temperature of the photons reaches about $3000 \mathrm{~K}\left(r(t) \sim 10^{-3}\right)$, the hydrogen atoms have a distance of about $a_{H}(t) \sim 10^{-3} \mathrm{~m}$ and are now partially ionized, i.e. we have free protons and electrons, both fermions, which appear in Figure 2 Since both particles are charged, they will emit and adsorb photons. From now on matter and radiation have approximately the same temperature $T(t) \sim 3 r^{-1}(t) K$. The initial coordinates are $a_{p}(t) / \star_{C, p} \sim 10^{13}, T / m_{p} c^{2}=$ $\left(\lambda_{C, p} / \lambda_{T, \nu}\right)^{\nu} \sim 10^{-10}$ ( i.e. $a_{p}(t) / \lambda_{T, 2_{p}} \sim 10^{8}$ ) for protons and $a_{e}(t) / \lambda_{C, e} \sim 10^{10}, T / m_{e} c^{2}=\left(\lambda_{C, e} / \lambda_{T, \nu}\right)^{\nu} \sim$ $10^{-7}$ ( i.e. $a_{e}(t) / \star_{T, 2} \sim 10^{6}$ ) for electrons, respectively. Thus their initial positions are in region (i) of Figure 1. As long as $a_{T} / \lambda_{C} \sim 10^{4} \ll a(t) / \star_{C}$, electrons and protons form initially an non-interacting non-relativistic classical plasma (compare Figure 2). Since $a(t) \sim r(T)$ and $T(t) \sim r^{-1}(t)$, the slope of the path under further contraction in the double logarithmic plot of Figure 2 is -1 and remains unchanged until we reach the relativistic region $T>m c^{2}$.

When the contraction parameter reaches $r(t) \sim 10^{-10}$, i.e temperatures reach about $3 \cdot 10^{10} \mathrm{~K}$, the mean particle distance is of the order of the Bohr radius.

When temperatures reach the rest energy of the electrons, i.e. crossing the bisecting line $T=m_{e} c^{2}$ of Figure 2, the electrons start to behave relativistically. For $T \gg m_{e} c^{2}$ electron-positron pairs are formed and leptons behave ultra-relativistically. For temperatures $T>10^{15} \mathrm{~K}$ and $a_{p} \sim 10^{-15} \mathrm{~m}$ hadrons decay into quarks and anti-quarks, which also behave ultra-relativistically. Also their mean distance is now determined by pair production. In addition gluons, i.e. the bosons which carry the interaction between quarks, have to be considered. Eventually the coordinates of all fundamental fermions and bosons reach the line $a \sim \lambda_{T, 1}$.

The pressure in all cases is that or ultra-relativistic fermions (3.23) or bosons (3.29), respectively. The total pressure is the sum of these contributions, i.e. it is proportional to the number of different fermions and bosons. 


\section{ADDITIONAL THERMODYNAMIC}

We can also use the results of the previous Chapters to determine the scaling behavior of the free energy $f(T, v)$ per particle which can be written as

$$
f(T, v)=-T \varphi_{\mathrm{F} / \mathrm{B}}\left(x_{\nu}\right)
$$

Differentiation with respect to $v=a^{d}$ gives

$$
-\frac{\partial f}{\partial v}=\frac{T \varphi_{\mathrm{F} / \mathrm{B}}^{\prime}\left(x_{\nu}\right) x_{\nu}}{v d}=p .
$$

Thus $\psi_{\mathrm{F} / \mathrm{B}}\left(x_{\nu}\right)=(1 / d) x_{\nu} \varphi_{\mathrm{F} / \mathrm{B}}^{\prime}\left(x_{\nu}\right)$. The entropy per particle $s$ follows from

$$
s=-\frac{\partial f}{\partial T}=\varphi_{\mathrm{F} / \mathrm{B}}\left(x_{\nu}\right)+\frac{1}{\nu} \varphi_{\mathrm{F} / \mathrm{B}}^{\prime}\left(x_{\nu}\right) x_{\nu}
$$

and hence we get for the energy per particle $u$

$$
u=f+T s=\frac{T}{\nu} \varphi_{\mathrm{F} / \mathrm{B}}^{\prime}\left(x_{\nu}\right) x_{\nu}=\frac{d}{\nu} p v
$$

which is the well known relation between the energy $u$ and $p v$ both for relativistic and non-relativistic particle in any dimension ${ }^{29}$. For the chemical potential $\mu$ we obtain from the Gibbs-Duham relation

$$
\mu=f+p v=T\left(\frac{1}{d} \varphi_{\mathrm{F} / \mathrm{B}}^{\prime}\left(x_{\nu}\right) x_{\nu}-\varphi_{\mathrm{F} / \mathrm{B}}\left(x_{\nu}\right)\right) .
$$

With our Ansätze used above we find at low temperatures $s=0$ for fermions and $\mu=0$ for bosons.

These expressions can be used to calculate additional thermodynamic quantities. The specific heat follows from (5.37) as

$$
c_{T}=\frac{\partial u}{\partial T}=\frac{d}{\nu} \frac{\partial}{\partial T}(p v)=\frac{d}{\nu} \frac{\partial}{\partial T}\left(T \psi_{\mathrm{F} / \mathrm{B}}\left(x_{\nu}\right)\right) .
$$

For fermions our Ansatz $\psi_{F}\left(x_{\nu}\right) \sim x^{-\nu}$, i.e. $f \sim E_{F}$, gives $c_{T}=0$ instead of the correct result $c_{T} \sim T / E_{F} \sim$ $x_{\nu}^{\nu}$. This is not surprising since we determined $v p \sim f$ from the condition that the free energy should go to a constant (the Fermi energy) for $T \rightarrow 0$.
To get the correct specific heat we have to add finite temperature corrections to $u$. Indeed, the low temperature corrections to energy of the Fermi gas result from the difference $\Delta$ of the Fermi distribution at non-zero and zero temperatures (the latter is the step function). These corrections are of the form ${ }^{32}$

$$
u=\int_{-\infty}^{\infty} d E g(E) E \Delta\left(\frac{E-\mu}{T}\right)
$$

where $\Delta(x)=\left(e^{x}+1\right)^{-1}-\theta(-x)$, and $g(E)$ is the density of states which is smooth at $E=\mu$. With $\Delta(x)=-\Delta(-x)$ it is clear that the low temperature expansion of $u$ includes only even powers in $T$. Since $E_{F}$ is the only energy scale, the low temperature expansion of the energy of the ideal Fermi gas is therefore of the form

$$
u \sim E_{F}\left(1+\frac{T^{2}}{E_{F}^{2}}+c_{2} \frac{T^{4}}{E_{F}^{4}}+\cdots\right)
$$

which is the Sommerfeld expansion. This gives for the specific heat $c_{T} \sim T / E_{F}{ }^{30}$. On the other hand, calculating the compressibility we get with $\psi_{F}\left(x_{\nu}\right) \sim x_{\nu}^{-\nu}$ immediately the correct result

$$
\kappa=-\frac{1}{V} \frac{\partial V}{\partial p}=\frac{d}{\nu+d} p^{-1}
$$

For bosons we obtain with $\psi_{B} \sim x^{d}$ the correct result for the specific heat ${ }^{33.34} c_{T} \sim \frac{\partial}{\partial T}\left(T x_{\nu}^{d}\right) \sim\left(T / T_{c}\right)^{d / \nu} \sim$ $\left(a / \lambda_{T, \nu}\right)^{d}$ and the compressibility diverges.

To conclude we have shown that the main properties of ideal quantum gases can be obtained from simple dimensional arguments and the Pauli principle, without resorting to statistical mechanics.

\section{Acknowledgment}

It is a pleasure to acknowledge helpful advice on the preparation of this paper by A. Glatz, C. Kiefer, J. Krug, E. Müller-Hartmann, A. Rosch, B. Rosenow, S. Scheidl, and D. Stauffer.
1 Most likely, some of the arguments presented in this paper are not new, but I have never encountered this formulation elsewhere.

2 Strictly speaking also quasi-particles exhibit a dispersion which we ignore here.

3 L. D. Landau and E. M. Lifshitz: "Statistical Physics, part 1", 7th ed., Akademie Verlag Berlin, chapter $\mathbf{7 1 .}$

4 Antiferromagnets are magnets with an opposite magnetization on two inter-penetrating sublattices. See e.g.L. D. Landau and E. M. Lifshitz: "Statistical Physics, part 2", 7th ed., Akademie Verlag Berlin, chapter $\mathbf{7 4 .}$
5 Reference ${ }^{4}$, chapter $\mathbf{7 0}$.

${ }^{6}$ P.W. Anderson, "Basic Notions of Condensed Matter Physics", Benjamin, Menlo Park, California, 1984.

7 D.O. Edwards and W.F. Sam, in "Progress in Low Temperature Physics", ed. by Brewer, Vol. VIIb, p.283, NorthHolland Publishing Company 1978.

8 E. H. Lieb and J Yngvason, Notices of the AMS, May 1998, p. 571 and references therein.

9 To get this relation one starts from the Ansatz for the pressure $p \sim T^{\alpha} a^{\beta} \gamma_{\nu}^{\delta}$ and compares the powers of the di- 
mensions of $M, L, t$ on both sides of this relations, which determines the exponents $\alpha, \beta, \delta$.

10 Thus we put $k_{B}=1,1$ Kelvin corresponds roughly to $10^{-4} \mathrm{eV}$

11 In our units $a_{T}=\frac{e^{2}}{T}=\frac{\star_{T, 2}^{2}}{a_{B}}$ and $\frac{a_{T}}{\star_{C}}=\frac{1}{137} \frac{\star_{T, 2}^{2}}{\star_{C}^{2}}$.

12 Trivially, any power of $x_{\nu}$ is also a solution.

13 Note that in the classical region $\lambda_{C}<\lambda_{T, \nu}<a$ the relevant thermal de Broglie length is $\lambda_{T, 2}$ whereas in the ultrarelativistic quantum region $a<\lambda_{T, \nu}<\lambda_{C}$ this role is played by $\lambda_{T, 1}$.

14 R. Friedberg, T.D. Lee and H.C. Renn, Annals of Physics 208, 149-215 (1997).

15 These results can be found, including the numerical prefactors, which remain undetermined in our approach, e.g. in Reference ${ }^{3}$, eq. (56.9)

16 Reference $^{3}$, eq. (61.7)

17 Refernce $^{3}$, eq. (45.5)

18 Considering the partition of two particles on two states $|x\rangle,|y\rangle$ of equal energy, classically there are 4 possibilities, $|x\rangle|x\rangle,|y\rangle|y\rangle,|x\rangle|y\rangle$ and $|y\rangle|x\rangle$. The probability that two particles are in the same state, say $|x\rangle$, is $1 / 4$. In quantum mechanics only the states $|x\rangle|x\rangle,|y\rangle|y\rangle$ and $\frac{1}{\sqrt{2}}(|x\rangle|y\rangle+$ $|y\rangle|x\rangle)$ exist, i.e. the probability for having two particles in the same state is $1 / 3$.

19 Reference ${ }^{3}$, eq. (56.15).

20 Reference ${ }^{3}$, eq. (61.4)

21 Reference ${ }^{3}$, eq. (57.7).

22 Reference $^{3}$, eq. (106.5)

23 Reference ${ }^{3}$, eq. (61.7)

24 Reference $^{3}$, eq. (101.2)

${ }^{25}$ Reference $^{3}$, eq. (63.16).

26 Reference ${ }^{3}$, eq. (62.9).

27 For particles with vanishing chemical potential like photons or quasi-particles there is no particle number conservation and hence no condensate, particles just disappear from the system.

28 Reference $^{3}$, eq. (62.4). We have chosen here the numerical prefactor of $T_{c, 2}$, which remains undetermined in our approach, such that a non-zero condensate appears first at $T_{c, 2}$.

29 Reference ${ }^{\frac{3}{}}$, compare eqs. (56.8), (61.4), (63.17).

30 Reference ${ }^{3}$, eq. (58.6).

31 A star of radius $R \leq R_{S}$ would be a black hole.

${ }^{32}$ Here we use a modest amount of statistical mechanics.

33 Reference ${ }^{3}$, eq. (62.6).

${ }^{34}$ Reference $^{3}$, eq. (63.15). 